\title{
Desarrollo de competencias científicas a partir de las propiedades de la materia*
}

Artículo de investigación

Fecha de Recepción: 10 diciembre 2017.

Fecha de Aprobación: 4 mayo 2018.

\author{
Adela del Pilar Suárez Mendoza** \\ Jenny Patricia Sánchez Ariza **** \\ Pilar Yadira Bastidas Velandia
}

\section{Resumen}

Esta propuesta corresponde a un estudio cualitativo, con diseño de Investigación Acción, desarrollada con 40 estudiantes del curso 6-2 del Colegio Isabel Valbuena Cifuentes del municipio de Vélez (Santander). El objetivo fue desarrollar las competencias científicas en estos estudiantes, empleando un método en el aprendizaje de las Ciencias Naturales, teniendo como base los estándares propuestos por el Ministerio de Educación Nacional (2004). En la primera fase de este estudio, se realizó la identificación de las competencias científicas de los estudiantes aplicando una prueba escrita, y un taller práctico. En la segunda fase, se aplicó una secuencia de aprendizaje; y, en la última fase, se evaluó el avance de los estudiantes, mediante la misma prueba escrita y otro taller práctico con los mismos parámetros del primero. Los resultados obtenidos fueron analizados, evidenciando que la población objeto de estudio se encontraban en un nivel incipiente, siendo escaso o nulo el desarrollo de cada competencia. Como conclusión, el método de enseñanza y aprendizaje de las Ciencias, implementado en esta investigación, constituyó la estrategia que permitió el desarrollo de las competencias científicas en los estudiantes.

Palabras clave: competencias, aprendizaje, ciencias naturales, investigación, enseñanza.
*Artículo de investigación científica y tecnológica.

** Colegio Isabel Valbuena Cifuentes, Vélez (SantanderColombia)

suapili@hotmail.com

$* * * *$ Colegio Isabel Valbuena

Cifuentes, Vélez (Santander-

Colombia)

jennypsa17@hotmail.com

**:**: Colegio Isabel

Valbuena Cifuentes, Vélez

(Santander-Colombia)

pilaryadira82@yahoo.es

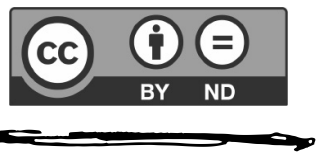




\section{Introducción}

Uno de los retos del docente, es orientar un área del saber en la cual los estudiantes desarrollen competencias y generen conocimiento, esto implica un conjunto de habilidades y actitudes que determinan la realización de una acción en un contexto determinado (Orrego \& Toro, 2014).

En la actualidad, los estudiantes presentan actitudes que muestran poco interés por la clase de Ciencias Naturales. Esta situación limita su desarrollo, como lo afirma Pozo \& Gómez (1998), "las actitudes constituyen una de las principales dificultades para la enseñanza y aprendizaje de las ciencias" (p. 34). Debido a esto, la falta de motivación es un impedimento en la enseñanza de las Ciencias Naturales,

Para muchos profesores de educación secundaria, sobre todo en la ESO, este es el principal problema al que se enfrentan. Los alumnos no están interesados en la ciencia, no quieren esforzarse ni estudiar y, por consiguiente, dado que aprender ciencias en una tarea intelectual, compleja y exigente, fracasan. (Pozo \& Gómez, 1998, p. 44)

Para muchos profesores de educación secundaria, sobre todo en la ESO, este es el principal problema al que se enfrentan. Los alumnos no están interesados en la ciencia, no quieren esforzarse ni estudiar $y$, por consiguiente, dado que aprender ciencias en una tarea intelectual, compleja y exigente, fracasan. (Pozo \&

Gómez,
La falta de motivación que hay en el Colegio Isabel Valbuena Cifuentes del municipio de Vélez, se presenta por la metodología empleada en la enseñanza de las ciencias, ya que tiende a ser muy tradicional. Como indica Bachelard (1948) "Los profesores de ciencias se imaginan que el espíritu comienza como una lección que siempre puede rehacerse una cultura perezosa repitiendo una clase, que puede hacerse comprender una demostración repitiéndola punto por punto" (p. 21), hacen falta estrategias en el aula de clase que permitan al estudiante explorar, indagar y experimentar, promoviendo el libre desarrollo de su creatividad y curiosidad innata, que es la que lo convierte en científico.

Al llegar a la básica media, presentan un bajo desempeño en el área de Ciencias Naturales, evidenciado en los resultados de las pruebas Saber 11, lo que perjudica su paso a la universidad, limitándolos a escoger carreras profesionales aisladas de las Ciencias, como se puede ver en el documento Estudiar Ciencias, publicado por la Universidad de los Andes, en el que Paula Luna, estudiante de Química, recopiló investigaciones que llevan a concluir que "a pesar de la buena apreciación que tienen los jóvenes de los beneficios de la ciencia y la tecnología, muy pocos la consideran una carrera científica como una opción profesional” (Luna, 2015, p. 90).

Ante esta situación, se planteó la pregunta ¿Cómo desarrollar las competencias científicas a partir de las propiedades de la materia: masa, volumen y densidad, en los estudiantes de curso $6 .^{\circ}$ del Colegio Isabel Valbuena Cifuentes?, teniendo en cuenta que el aprendizaje de las Ciencias Naturales es esencial en los primeros años de básica secundaria. Esta investigación toma como grupo de trabajo, estudiantes del grado sexto, pues es en esta edad, según Piaget, donde el niño adquiere un pensamiento más científico, 
edad llamada estadio de operaciones formales, que va desde los 11 años a la edad adulta (Woolfolk, 2010). El fin es motivar a los estudiantes mediante una metodología nueva en el plan de área de Ciencias Naturales de la institución, dejando de lado la metodología tradicional con la que se ha venido trabajando, de manera que, desde temprana edad, se garantice que las ciencias tengan algún significado real para los estudiantes, sea algo útil y divertido. También es importante que la educación científica le permita al estudiante sentirse a gusto con la ciencia, como aspecto importante de la experiencia humana en el pasado, presente y futuro, de igual forma, verla como medio para mejorar la calidad de vida (Harlen, 2007).

Es un estudio cualitativo, con diseño de Investigación Acción, que tiene como objetivo principal, desarrollar las competencias científicas a partir de las propiedades de la materia (masa, volumen y densidad). Para alcanzar dicho objetivo, se plantearon tres fases de desarrollo: en la primera fase se buscó identificar las competencias científicas en los estudiantes; en la segunda, aplicar una secuencia de actividades, y es aquí donde se implementa el método que Harlen (2007) propone en su libro titulado $L a$ enseñanza de las Ciencias Naturales, que consiste en que el estudiante realice actividades experimentales para lograr el desarrollo de dichas competencias. En la última fase de esta investigación, se evaluó el avance que presentaron los estudiantes luego de aplicada la secuencia de actividades de aprendizaje.
De esta forma, también se quiere resaltar el lenguaje, pues es una pieza fundamental en el desarrollo cognoscitivo del niño, como lo dice Vigotsky (citado en Woolfolk, 2010), ya que ofrece un medio para expresar ideas y formular preguntas, así como para concebir las categorías y los conceptos del pensamiento. Dado el caso que el Instituto Colombiano para la Evaluación de la Educación (ICFES, 2007) en su examen mide solo tres de las competencias científicas, este proyecto tiene como elemento innovador el desarrollo de la competencia denominada comunicación, que busca evaluar otras como la obtención de conclusiones y la comunicación.

\section{Desarrollo de competencias científicas}

El papel del docente no consiste únicamenteen transmitirconocimientos, pues es el facilitador del aprendizaje de los estudiantes, por lo tanto, una de sus labores es involucrarlos en la construcción del conocimiento a través de estrategias que motiven la curiosidad y el deseo de aprender más. Al respecto, Pozo \& Gómez (2013) afirman:

La enseñanza de la ciencia se muestra habitualmente ineficaz para lograr los profundos cambios no solo conceptuales, sino también actitudinales y procedimentales, que requiere la transición del conocimiento cotidiano al conocimiento científico. De hecho, puede decirse que la enseñanza tradicional de la ciencia no consigue promover estos cambios en los alumnos. (p. 129) 
Una de las metas fundamentales de la formación en ciencias es procurar que los y las estudiantes se aproximen progresivamente al conocimiento científico, tomando como punto de partida su conocimiento

"natural" del mundo y fomentando en ellos una postura crítica que responda a un proceso de análisis y reflexión.
El docente de Ciencias Naturales puede proporcionar herramientas que contribuyan al desarrollo de habilidades cognitivas del estudiante, favoreciendo el saber hacer del mismo en un contexto determinado, pero también la preparación para las pruebas de Estado. Por otro lado, el Ministerio de Educación Nacional MEN (2004) plantea:

Una de las metas fundamentales de la formación en ciencias es procurar que los y las estudiantes se aproximen progresivamente al conocimiento científico, tomando como punto de partida su conocimiento "natural" del mundo y fomentando en ellos una postura crítica que responda a un proceso de análisis y reflexión. (p. 104)

También, refiere que las competencias son el conjunto de conocimientos, habilidades y actitudes que el sujeto utiliza en un contexto determinado, demostrando un desempeño adecuado.

De acuerdo con los planteamientos del MEN, mencionados anteriormente, el Instituto Colombiano para la Evaluación de la Educación, valora tres competencias fundamentales en el área de Ciencias Naturales, que son: la identificación, indagación y la explicación (ICFES et al., 2007). A continuación, se explican:

1. Identificar. Capacidad para reconocer y diferenciar fenómenos y representaciones (se entiende por representaciones las nociones, los conceptos, las teorías, los modelos $\mathrm{y}$, en general, las imágenes que nos formamos de los fenómenos) a partir del conocimiento adquirido.
2. Indagar. Capacidad para seleccionar, organizar e interpretar información relevante y para diseñar y elegir procedimientos adecuados con el fin de dar respuesta a una pregunta.

3. Explicar. Capacidad para seleccionar y comprender argumentos y representaciones adecuados para dar razón de fenómenos. (pp. 3334)

Estas competencias se relacionan en su conjunto con las destrezas de procedimiento señaladas por Harlen (2007), ya que contribuyen al desarrollo de habilidades de pensamiento del niño, a través de procedimientos que le permiten obtener información de una situación o fenómeno, empleando las pruebas adecuadas. Las destrezas de procedimiento que son fundamentales para la enseñanza de las Ciencias, son: "observación, elaboración de hipótesis, predicción, investigación, derivación de conclusiones, comunicación" (Harlen, 2007, p. 73).

En la figura 1 se relacionan las competencias propuestas por el ICFES que son tres y que abarcan en su definición cinco de las seis destrezas de procedimiento propuestas por Harlen (2007).

En esta figura, se realiza la correlación entre las competencias que evalúa el ICFES (2007) y las destrezas de procedimiento propuestas por Harlen (2007), se destacan estas dos fuentes, que son las que principalmente se tuvieron en cuenta para el desarrollo del objetivo principal de esta investigación. La sexta destreza de 
Figura 1. Relación de las competencias ICFES (2007) y Harlen (2007).

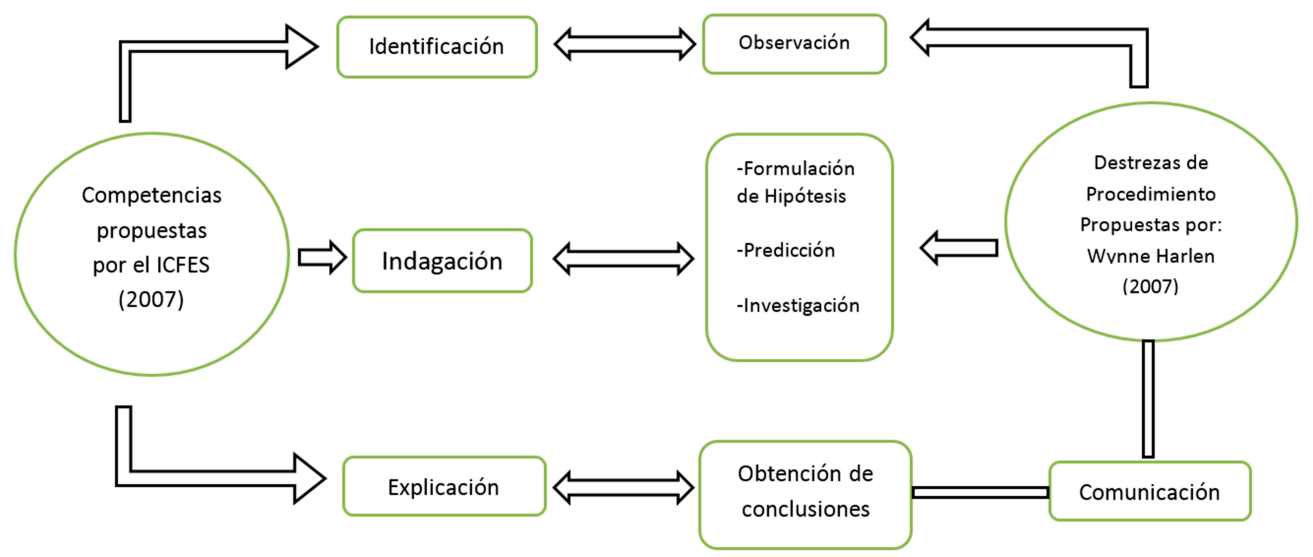

Fuente: Autoras del trabajo

procedimiento, que es la competencia de comunicación, el ICFES no la evalúa. La competencia explicación fomenta en el estudiante una actitud analítica que le posibilita establecer la validez o coherencia de una afirmación o de un argumento (ICFES et al., 2007), pero no le permite comunicarse. Por esta razón, la comunicación no tiene una correlación directa con las competencias propuestas por el ICFES.

También es importante señalar que, de acuerdo con las etapas del desarrollo del pensamiento del niño propuestas por Piaget, quien describió la evolución del desarrollo cognitivo de éste, la población objeto de este estudio se ubica en la etapa de operaciones formales (de 11 años a la edad adulta) en la cual es capaz de resolver problemas abstractos de forma lógica (Samacá, 2016). Su pensamiento se vuelve más científico (Woolfolk, 2010, p. 34).

De esta manera, esta investigación pretendió implementar un método de enseñanza de la ciencia en el aula de clase, mediante el desarrollo de competencias propias de esta área y en una de las poblaciones con las cuales se pudo tener contacto por corresponder al área y nivel de desempeño de las docentes investigadoras.

\section{Contribuciones metodológicas}

La investigación realizada es de tipo cualitativo, la cual requiere de un sujeto $y$ un investigador que interactúan partiendo de una situación problema que permite al investigador conocer algo sobre este sujeto. Este método se considera la mejor herramienta para adquirir la información necesaria, puesto que el docente debe participar con el grupo de estudiantes que se está investigando (Sandoval, 2002).

La investigación en cuestión, tiene un enfoque de Investigación Acción, ya que se relaciona, según Elliott (2000), con los problemas prácticos cotidianos 
experimentados por los profesores, en vez de los "problemas teóricos" definidos por los investigadores puros en el entorno de una disciplina del saber. Es decir, que las acciones van encaminadas a modificar la situación inicial, con el objeto de lograr una comprensión profunda de los problemas del aula de clase.

Siguiendo a Elliott (2000), el propósito de la investigación-acción consiste en profundizar la comprensión del profesor (diagnóstico) de su problema. Dicha comprensión no impone una respuesta específica, sino que indica, de manera general, la respuesta más adecuada. Esta respuesta debe surgir de la comprensión o diagnóstico profundizado por el profesor.

El propósito central de la investigación acción es la transformación de la situación-problema que afecta a la población involucrada, en el caso de ésta, no se trata de estudiar problemas de interés científico $o$ cuestiones que preocupan a un grupo de investigadores, sino los concernientes a la práctica educativa que se consideran importantes porque tienen que ver con la vida y desarrollo intelectual de los estudiantes. En la investigación acción, el docente se convierte en un investigador de su práctica pedagógica mediante una reflexión constante, con miras a la transformación permanente de esta. Por lo tanto, el presente estudio reflexiona sobre esta práctica reconstruyéndola y planeando los cambios a través de acciones transformadoras, finalizando con la evaluación de estos cambios.
El estudio se desarrolló en el municipio de Vélez (Santander, Colombia), en la Institución Educativa Colegio Isabel Valbuena Cifuentes, ubicada en la zona urbana del municipio. La muestra fue la totalidad de los estudiantes del curso 6-2, con 40 estudiantes de edades que oscilan entre 11 y 12 años, con estratos 1 y 2 , de la jornada de la mañana, vinculados directamente con la enseñanza de la asignatura ciencias naturales.

En tal sentido, se planteó un estudio que permitió aproximar el desarrollo de las competencias científicas en los estudiantes de grado sexto curso 6-2, aplicando una secuencia de aprendizaje; $\mathrm{y}$, finalmente, evaluar el avance en estos estudiantes.

\section{Instrumentos utilizados en la investigación.}

Los instrumentos responden a un proceso de investigación acción, la cual, según Elliott (2000), "interpreta lo que ocurre desde el punto de vista de quienes actúan e interactúan en la situación problema”, en este caso, profesores y alumnos.

Para desarrollar el primer objetivo de esta investigación, se aplicaron dos instrumentos: el primero fue una prueba escrita que constaba de 15 preguntas seleccionadas de los cuadernillos de Pruebas Saber Segunda Edición Ciencias Naturales grado 5, clasificando las preguntas con base en los Estándares Básicos de Competencia de grado 5. Los estándares de Ciencias Naturales tienen un énfasis en competencias, buscando así el 
desarrollo de las habilidades y actitudes científicas por parte de los estudiantes (ICFES et al., 2007).

Para el análisis de los resultados de la prueba escrita aplicada en esta fase, se valoraron los desempeños de los estudiantes, articulándolos con el sistema de evaluación de la Institución, basados en el Decreto 1290 del 2009, por el cual se reglamenta la evaluación del aprendizaje de los estudiantes de los niveles de educación básica y media (MEN, 2009).

Tabla 1. Categorización de desempeño de los estudiantes en la prueba escrita.

\begin{tabular}{cc}
\hline Desempeños & $\begin{array}{c}\text { Total, de preguntas } \\
\text { acertadas }\end{array}$ \\
\hline Bajo & $1-6$ \\
\hline Básico & $7-10$ \\
\hline Alto & $11-14$ \\
\hline Superior & 15 \\
\hline
\end{tabular}

Fuente: Decreto 1290 (MEN, 2009, p. 2).

Desde el área de Ciencias Naturales, el ICFES (2007) define siete competencias quecorresponden a lacapacidad deacción que se consideran relevantes: "identificar, indagar, explicar, comunicar, trabajar en equipo, disposición para aceptar la naturaleza abierta parcial y cambiante del conocimiento, y disposición para reconocer la dimensión social del conocimiento y para asumirla responsablemente", pero, solo tres de ellas (identificar, indagar, explicar) son evaluadas por este tipo de pruebas. Por lo tanto, se diseñó también un taller práctico como segundo instrumento basado y modificado en una propuesta de Harlen (2007, pp. 187-189) y de esta manera identificar el estado inicial del desarrollo de las competencias científicas de los estudiantes.

Harlen (2007) afirma que las competencias científicas deben ser desarrolladas y evaluadas de forma práctica. Por lo tanto, con este taller se realizó un diagnóstico de estas competencias en los estudiantes, que son: observación, formulación de hipótesis, predicción, investigación, conclusión y comunicación. Para esto, se tomaron los criterios establecidos por Harlen (2007) para valorar el aprendizaje de las ciencias y se asignaron los números del uno al tres de acuerdo con el nivel de desarrollo, siendo 1 para el más bajo y 3 para el más alto. Así como lo describe la siguiente tabla:

Tabla 2. Criterios de valoración de las competencias científicas.

\begin{tabular}{llc}
\hline \multirow{2}{*}{ Competencia } & \multicolumn{1}{c}{ Criterio } & $\begin{array}{c}\text { Nivel de } \\
\text { desarrollo }\end{array}$ \\
\hline \multirow{3}{*}{ Observación } & $\begin{array}{l}\text { No da ningún indicio de que se dé cuenta de cosas no habituales } \\
\text { ni de detalles de cosas conocidas, a menos que se le señalen. }\end{array}$ & 1 \\
\cline { 2 - 3 } & $\begin{array}{l}\text { Hace observaciones utilizando diversos sentidos, pero hay que } \\
\text { ayudarle a descubrir pautas y secuencias en lo que no descubre. }\end{array}$ & 2 \\
\cline { 2 - 3 } & $\begin{array}{l}\text { Utiliza pautas y relaciones en observaciones de carácter general, } \\
\text { centrando posteriormente la observación en lo que sea relevante } \\
\text { para un determinado problema o investigación. }\end{array}$ & 3 \\
\hline
\end{tabular}

Harlen (2007) afirma que las competencias científicas deben ser desarrolladas y evaluadas de forma práctica. 


\begin{tabular}{|c|c|c|}
\hline \multirow{3}{*}{ Hipótesis } & No sugiere hipótesis para explicar las observaciones iniciales. & 1 \\
\hline & $\begin{array}{l}\text { Sugiere hipótesis para explicar observaciones iniciales, aunque } \\
\text { hay que ayudarle a utilizar la información o las ideas previas } \\
\text { para explicar la nueva experiencia. }\end{array}$ & 2 \\
\hline & $\begin{array}{l}\text { Sugiere hipótesis para explicar observaciones iniciales } \\
\text { utilizando la información o ideas previas para explicar la nueva } \\
\text { experiencia. }\end{array}$ & 3 \\
\hline \multirow{3}{*}{ Predicción } & $\begin{array}{l}\text { No hace predicciones basadas en las pautas observadas, en las } \\
\text { hipótesis o en su experiencia previa. }\end{array}$ & 1 \\
\hline & $\begin{array}{l}\text { Hace predicciones basadas en las pautas observadas y en las } \\
\text { hipótesis, aunque hay que ayudarle a utilizar la información o } \\
\text { las ideas previas para explicar la nueva experiencia. }\end{array}$ & 2 \\
\hline & $\begin{array}{l}\text { Hace predicciones basadas en las pautas observadas, en las } \\
\text { hipótesis o en su experiencia previa para explicar la nueva } \\
\text { experiencia. }\end{array}$ & 3 \\
\hline \multirow{3}{*}{ Investigación } & $\begin{array}{l}\text { No planea ni desarrolla una investigación sencilla en la } \\
\text { experiencia cotidiana mediante las acciones adecuadas a lo que } \\
\text { haya que descubrir. }\end{array}$ & 1 \\
\hline & $\begin{array}{l}\text { Planea y desarrolla una investigación sencilla en la experiencia } \\
\text { cotidiana, aunque hay que ayudarle a desarrollarla utilizando la } \\
\text { información y elegir las acciones adecuadas a lo que haya que } \\
\text { descubrir. }\end{array}$ & 2 \\
\hline & $\begin{array}{l}\text { Planea y desarrolla una investigación sencilla en la experiencia } \\
\text { cotidiana mediante las acciones adecuadas a lo que haya que } \\
\text { descubrir. }\end{array}$ & 3 \\
\hline \multirow{3}{*}{$\begin{array}{l}\text { Obtención de } \\
\text { conclusiones }\end{array}$} & $\begin{array}{l}\text { No extrae conclusiones para la investigación, basada en las } \\
\text { observaciones efectuadas o en las informaciones conseguidas. }\end{array}$ & 1 \\
\hline & $\begin{array}{l}\text { Extrae conclusiones generales para la investigación, basada en } \\
\text { las observaciones efectuadas o en las informaciones conseguidas, } \\
\text { pero hay que ayudarle a descubrir conclusiones relevantes. }\end{array}$ & 2 \\
\hline & $\begin{array}{l}\text { Extrae conclusiones relevantes y justificadas para la } \\
\text { investigación, basada en las observaciones efectuadas o en las } \\
\text { informaciones conseguidas. }\end{array}$ & 3 \\
\hline \multirow{3}{*}{ Comunicación } & $\begin{array}{l}\text { No explica claramente, en forma oral o escrita, los hallazgos o } \\
\text { descubrimientos de la investigación. }\end{array}$ & 1 \\
\hline & $\begin{array}{l}\text { Explica claramente, de forma oral o por escrito, la investigación, } \\
\text { aunque hay que ayudarle a interpretar y resumir los hallazgos. }\end{array}$ & 2 \\
\hline & $\begin{array}{l}\text { Explica con claridad, de forma oral o por escrito, la investigación, } \\
\text { utilizando gráficos y tablas para resumir los hallazgos. }\end{array}$ & 3 \\
\hline
\end{tabular}

Fuente: adaptación de Harlen (2007, pp. 172-180).

Los criterios antes mencionados, definen los momentos de desarrollo de cada competencia científica que esta investigación aborda, permitiendo a las investigadoras dirigir la atención a las conductas significativas en el desarrollo de las mismas. De esta manera, la información obtenida se hace más eficiente, al saber lo que se busca; es necesario definir los criterios antes de realizar las observaciones. 
Para la ejecución del segundo objetivo, se desarrolló la secuencia de talleres, a partir del modelo del proceso de aprendizaje planteado por Harlen (2007), el cual tiene una función predictiva, ya que "el punto de partida del modelo es la evidencia de que las ideas derivadas de las experiencias precedentes se utilizan para tratar de dar sentido a la experiencia nueva" (p. 40). El tema que se tuvo como base para la ejecución de este objetivo, fue las propiedades de la materia (masa, volumen, densidad, tema seleccionado teniendo en cuenta los estándares de competencia de grado 6) con el propósito de desarrollar las competencias científicas.

Los talleres fueron sujetos a juicio de expertos antes de su aplicación y, de esta manera, contrastar la validez del contenido de cada uno. "La evidencia de la validez de criterio se produce al correlacionar las puntuaciones de los participantes, obtenidas por medio del instrumento con sus valores logrados en el criterio" (Hernández, Fernández \& Baptista, 2010, p. 304).

En esta fase de la investigación, se hizo el seguimiento y registro mediante el diario de campo, el cual se diseñó a partir de Porlán \& Martín (1997) quienes afirman que este es un recurso metodológico núcleo de todo proceso, que permite establecer vínculos significativos entre la teoría, el programa y la práctica (p. 21). El seguimiento de cada estudiante se realizó mediante un modelo de diario de campo en el que se describe el objetivo de la sesión y lo que se observa durante el desarrollo de la clase, lo cual permitió realizar un análisis descriptivo y reflexivo de cada una de las actividades realizadas con los estudiantes; para, de esta forma, realizar los ajustes pertinentes durante el proceso, como por ejemplo: la búsqueda de espacios apropiados para la aplicación de talleres, la organización del trabajo grupal o individual de los estudiantes, la distribución del trabajo, de manera que unos grupos realizaran algunas actividades mientras que los otros realizaban otras, debido a los materiales insuficientes, la estructuración de actividades que beneficiaran el desarrollo de cada una de las competencias, la oportunidad de escuchar, de forma individual o grupal, comunicación de ideas o conclusiones, la planificación de los espacios y del tiempo para el desarrollo de cada competencia y para la explicación de temas como método de refuerzo, la consignación de apuntes necesarios y revisión de tareas adicionales realizadas en casa. Así, se realizó la observación directa del proceso de desarrollo de las competencias en cada estudiante, teniendo en cuenta el criterio para evaluar las técnicas de procedimiento en niños de 11 y 12 años en la enseñanza de las ciencias propuesta por Harlen (2007).

Por medio de este diario, se realizó una observación cualitativa, ya que no es solo contemplación ("sentarse a ver el mundo y tomar notas”); implicó mirar a profundidad situaciones sociales y mantener una reflexión permanente (Hernández, Fernández \& Baptista, 2010). 
Finalmente, para evaluar el avance en el desarrollo de las competencias científicas de los estudiantes (propuesto en el tercer objetivo de esta investigación), se aplicó el mismo instrumento aplicado en la fase inicial seleccionado de los Cuadernillos de Pruebas Saber de Ciencias Naturales grado 5 y un nuevo taller práctico que reunía las temáticas de este estudio (masa, volumen y densidad) sujeto también a juicio de expertos. Además, se tuvo en cuenta los mismos criterios de valoración del aprendizaje propuestos por Harlen (2007) y la misma codificación relacionada en la tabla 2.

\section{Resultados}

Para presentar los resultados de este proceso, se analiza cada una de las fases de la investigación. En forma general, se muestra que, al iniciar el estudio, los 40 estudiantes pertenecientes a la población investigada, presentaron un bajo nivel en el desarrollo de las seis competencias científicas evaluadas, después de aplicar la secuencia de aprendizaje propuesta y hacer la evaluación, se observa que la totalidad de la población avanzó gradualmente en el nivel de cada competencia. A continuación, se muestran detalladamente los resultados de cada fase:

\section{Prueba escrita aplicada en la primera y última fase de la investigación.}

Los resultados de la prueba escrita que se aplicó a los 40 estudiantes se muestran en la siguiente tabla en la cual se valoran sus desempeños, como se mencionó anteriormente, articulándolos con el sistema de evaluación de la Institución, basados en el Decreto 1290 del 2009, por el cual se reglamenta la evaluación del aprendizaje de los estudiantes de los niveles de educación básica y media (MEN, 2009).

Tabla 3. Análisis de resultados. Prueba escrita inicial.

\begin{tabular}{ccc}
\hline Desempeños & Total de preguntas acertadas & $\mathrm{N}^{\circ}$ de estudiantes \\
\hline Bajo & $1-6$ & 40 \\
\hline Básico & $7-10$ & 0 \\
\hline Alto & $11-14$ & 0 \\
\hline Superior & 15 & 0 \\
\hline
\end{tabular}

Fuente: adaptación Decreto 1290 (MEN, 2009, p. 2).

Con la tabla 3 , se evidencia que la totalidad de los estudiantes del grado 6-2 contestaron de 1 a 6 preguntas en forma acertada, por lo tanto, se encontraban en un desempeño académico bajo, según la escala de valoración adoptada por la Institución. A continuación, se muestran los resultados de la misma prueba escrita aplicada en la fase final de esta investigación y para su valoración se tuvieron en cuenta los mismos parámetros de la prueba inicial. 
Tabla 4. Análisis de resultados prueba escrita final

\begin{tabular}{ccc}
\hline Desempeños & Total de preguntas acertadas & $\mathrm{N}^{\circ}$ de estudiantes \\
\hline Bajo & $1-6$ & 4 \\
\hline Básico & $7-10$ & 15 \\
\hline Alto & $11-14$ & 16 \\
\hline Superior & 15 & 4 \\
\hline
\end{tabular}

Fuente: Decreto 1290 (MEN, 2009, p. 2).

Los resultados de prueba escrita aplicada en la fase final, se analizaron en la tabla 4, en la cual se muestra el avance académico de los estudiantes, pues al comparar los resultados de la fase inicial en la cual la totalidad de los estudiantes se encontraron en el desempeño bajo, en esta fase se destaca que solo 4 estudiantes siguen en este nivel de desempeño, 15 en el nivel de desempeño básico, 16 en el nivel de desempeño alto y 4 lograron el desempeño superior, para un total de 39 estudiantes, ya que en la última fase de este estudio se contó con este número porque un estudiante se retiró del Colegio, debido a traslado de domicilio familiar.

\section{Resultados de los dos talleres prácticos aplicados en la primera y última fase de la investigación.}

Para presentar los resultados, se va a tener en cuenta la primera fase de este estudio, cuyo objetivo era la identificación del estado de cada una de las competencias científicas que se pretendían desarrollar en los estudiantes; $y$, de esta manera, se establece un comparativo con respecto a la última fase, cuyo objetivo fue evaluar el avance de dichas competencias luego de aplicar una secuencia de talleres correspondientes a la segunda fase de la investigación.

En la tabla 5 se relacionan los resultados mencionados anteriormente:

Tabla 5. Análisis de resultados de talleres prácticos de la etapa inicial y final

\begin{tabular}{c|c|c|c}
\hline $\begin{array}{c}\text { Competencia } \\
\text { Científica }\end{array}$ & Nivel de desarrollo & $\begin{array}{c}\mathrm{N}^{\circ} \text { de estudiantes: } \\
40 \text { fase inicial }\end{array}$ & $\begin{array}{c}\mathrm{N}^{\circ} \text { de estudiantes: } \\
39 \text { fase final }\end{array}$ \\
\hline \multirow{3}{*}{ Observación } & 1 & 13 & 0 \\
& 2 & 27 & 6 \\
\hline \multirow{3}{*}{ Hipótesis } & 3 & 0 & 33 \\
& 2 & 39 & 35 \\
& 3 & 1 & 4 \\
\hline \multirow{3}{*}{ Predicción } & 1 & 0 & 0 \\
& 2 & 36 & 0 \\
\hline
\end{tabular}




\begin{tabular}{c|c|c|c}
\hline \multirow{3}{*}{ Investigación } & 1 & 38 & 1 \\
& 2 & 2 & 27 \\
\hline \multirow{2}{*}{ Obtención de } & 3 & 0 & 11 \\
conclusiones & 1 & 34 & 25 \\
& 3 & 6 & 13 \\
\hline \multirow{2}{*}{ Comunicación } & 1 & 0 & 1 \\
& 2 & 36 & 24 \\
\end{tabular}

Fuente: Autoras del Trabajo

En la tabla 5, se tomaron las competencias científicas del libro Enseñanza y aprendizaje de las Ciencias de Harlen (2007, pp. 172,180) y se elaboró un paralelo del número de estudiantes y el avance de acuerdo con el criterio de valoración, tanto en la fase inicial como en la final de este estudio.

Es necesario precisar que el último taller práctico aplicado a los estudiantes, no correspondió al primer taller estructurado para identificar las competencias científicas en la fase inicial, ya que este último atendió a una secuencia de aprendizaje, producto de los talleres aplicados en el segundo objetivo de este estudio; además, el nivel de complejidad fue mayor en el último taller, el cual reunió los tres temas estratégicos de esta investigación (masa, volumen y densidad) y, de igual forma, evaluó el avance de cada una de las competencias científicas de los estudiantes.

La comparación de los resultados de la fase de inicial y la fase final de este estudio correspondiente al desarrollo de competencias científicas de los estudiantes, permitió el siguiente análisis:
En primer lugar, los estudiantes demostraron un avance significativo en cada una de las competencias científicas, ya que pasaron de un nivel de desarrollo 1 a un nivel de desarrollo 2 o 3, como se muestra en la tabla 5. En la etapa final, un estudiante ya no hizo parte de este estudio en razón a un traslado de domicilio, por lo tanto, la población quedó conformada por 39 niños.

La competencia en la que se evidencia un avance total de los estudiantes de la escala 1 a la 3 , es la de elaboración de predicciones, ya que esta se desarrolla con base en las experiencias y presaberes de los niños, los cuales se van enriqueciendo a medida que se iba desarrollando la secuencia de aprendizaje, y teniendo en cuenta que los conocimientos y conceptos de ciencias naturales no son desarticulados sino secuenciales. Otra competencia en la que demostraron un gran avance fue en la de observación en la cual, 33 estudiantes pasaron a un nivel 3 , destacando que esta competencia constituye una de las principales por ser el inicio de todo quehacer científico.

En las competencias de investigación, conclusión y comunicación, un poco 
más de la mitad del grupo de estudiantes pasaron al nivel 2 en cada una de estas, y entre 11 y 14 estudiantes lograron llegar a un nivel 3 de desarrollo de estas competencias. Estos resultados son satisfactorios en este estudio, ya que el avance en estas tres competencias exige un mayor esfuerzo cognitivo por parte de los estudiantes.

Por último, hubo 1 estudiante que demostró avance en las competencias: observación, predicción e hipótesis, pasando de un nivel 1 al 2; y en las otras tres competencias: investigación, conclusión y comunicación, continuó en un nivel 1 pudiendo deberse a factores familiares y psicológicos que influyeron en este proceso. No obstante, las docentes investigadoras centraron también sus esfuerzos para colaborar y animar al niño hacia el desarrollo de estas competencias y brindaron apoyo moral en cada etapa de este proceso.

\section{Conclusiones}

Al realizar la identificación de cada una de las competencias científicas en la prueba escrita y en el taller práctico, se evidenció que la totalidad de la población objeto de estudio se ubicó en un criterio de valoración bajo. Este diagnóstico reflejó la necesidad de aplicar una metodología de enseñanza de las ciencias en el aula de clase para favorecer un conjunto de habilidades de pensamiento, en este caso, las competencias científicas propias de esta área; y, de esta forma, contribuir en el mejoramiento del desempeño académico de los estudiantes.
El método de enseñanza y aprendizaje de las Ciencias, implementado en la secuencia de aprendizaje (correspondiente a la ejecución del segundo objetivo específico de esta investigación), constituyó la estrategia que permitió el avance en el desarrollo de las competencias científicas. Cada uno de los talleres aplicados en dicha secuencia de aprendizaje, se estructuraron graduando la complejidad para su desarrollo, es decir, de un grado menor de complejidad a un grado mayor, de tal manera que los estudiantes comprendieran en qué consistía cada competencia y demostraran cada vez una mayor autonomía en el desarrollo de los mismos.

En cuanto a la evaluación de las competencias científicas correspondiente al tercer objetivo específico de esta investigación, se logró un avance sustancial en cada una de estas, ya que toda la población objeto de estudio ascendió de un criterio de valoración bajo a uno intermedio o alto en cada una de estas. A pesar de algunas dificultades cognitivas que presentaron algunos estudiantes (problemas de atención y lentitud al procesar la información), estos demostraron avances satisfactorios en el desarrollo de las competencias científicas.

Esta investigación tuvo como elemento innovador, potenciar la competencia de comunicación en los estudiantes. Se evidenció un avance significativo en cuanto a esta competencia, pues en la fase inicial la mayoría de los estudiantes no expresaban sus ideas de forma oral ni escrita, pero con el
Al realizar la identificación de cada una de las competencias científicas en la prueba escrita y en el taller práctico, se evidenció que la totalidad de la población objeto de estudio se ubicó en un criterio de valoración bajo. 
desarrollo de la propuesta se logró que ellos elaboraran su propio material de apoyo para comunicar los resultados y conclusiones de sus propios trabajos.

El método de enseñanza aprendizaje de las ciencias empleado en esta investigación, no solo motivó a los niños al aprendizaje de estas, sino que se favoreció el avance de las competencias científicas y el desempeño académico en esta área, de cada uno de estos estudiantes.

\section{Referencias}

Bachelard, G. (1948). La formación del espíritu científico. México: Siglo XXI Editores.

Elliott, J. (2000). La investigación-acción en educación. Madrid: Ediciones Morata, S. L.

Harlen, W. (2007). Enseñanza y Aprendizaje de las Ciencias. Madrid, España: Morata.

Hernández, R., Fernández, C., \& Baptista, P. (2010). Metodología de la Investigación. México: McGraw-Hill.

ICFES, Toro Baquero, J., Reyes Blandón, C., Martínez, R., Castelblanco, Y., Cárdenas, F., . . . Hernández, C. A. (2007, mayo). Fundamentación conceptual área de ciencias naturales. Recuperado de http://www.colombiaaprende.edu. co/html/competencias/1746/articles-335459_pdf_2.pdf

Luna, P. C. (2015). Ingreso y permanencia: ¿̇por qué los jóvenes colombianos no quieren estudiar ciencias, tecnología y matemáticas? Recuperado el 26 de agosto de 2016, de http://hipotesis.uniandes.edu.co/hipotesis/images/stories/ed18pdf/ Estudiar-ciencias-18.pdf

MEN. (2004). Estándares básicos de competencias en ciencias sociales y ciencias naturales. Recuperado dewww.colombiaaprende.edu.co/html/mediateca/1607/ articles-167860_archivo.pdf

MEN. (2009). Decreto 1290. Recuperado de http://www.mineducacion.gov. co/1621/articles-187765_archivo_pdf_decreto_1290.pdf

Orrego, J., \& Toro, L. (2014). Relaciones vitales: el aula como escenario permanente de investigación. Praxis \& Saber, 5(10), 121-139. https: // doi. org/10.19053/22160159.3025

Porlán, R. \& Martín, J. (1997). El diario del profesor. Un recurso para la investigación en el aula. Recuperado de: https://profesorailianartiles.files.wordpress. com/2013/03/diario-del-maestro.pdf 
Pozo, J. \& Gómez, M. (1998). Aprender y enseñar ciencia. Del conocimiento cotidiano al conocimiento científico. Madrid: Ediciones Morata.

Sandoval, C. (2002). Investigación Cualitativa. Bogotá: ARFO Editores e Impresores Ltda.

Samacá, I. (2016). El espíritu científico en la primera infancia. Praxis \& Saber, 7(13), 89-106. https://doi.org/10.19053/22160159.4167

Woolfolk, A. (2010). Psicología Educativa. México: Pearson Educación. 